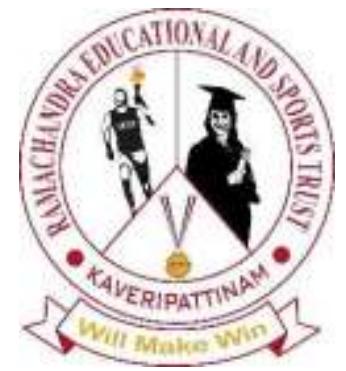

Recent trends in Management and Commerce

Vol: 2(3), 2021

REST Publisher

ISBN: 978-81-936097-6-7

Website: http://restpublisher.com/book-series/rmc/

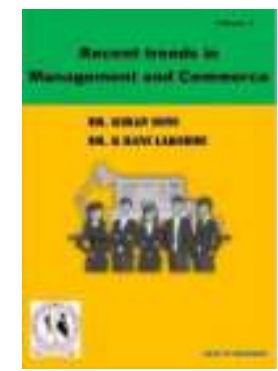

\title{
A Study on Climate Change with Mayfly Algorithm Optimization
}

\author{
M. Amudha, M. Ramachandran, Chinnasami Sivaji, R. Gayathri \\ REST Labs, Kaveripattinam, Krishnagiri, Tamil Nadu, India. \\ Email: chinnasami@ restlabs.in
}

\begin{abstract}
Mayfly algorithm (MA) is an optimal way to achieve this goal. It is a newly developed algorithm that combines the key benefits of PSO, GA and FA, resulting in a hybrid of PSO-GA-FA. Compared with the other seven transformation methods, the performance evaluation performed using the CEC test operations demonstrates the dominance of MA in terms of accumulation rate and velocity. Although this requires rapid integration, it is not yet used in many engineering optimization fields, so we propose to use it for antenna optimization tasks. Predictions for the next century 15 to $95 \mathrm{Cm}$, the $50 \mathrm{~cm}$ 'best estimate' indicates a significant increase in sea level rise over the last century, with records from both the Atlantic and the Pacific in the Northern Hemisphere showing sea increase. However, in most cases, of some sites This condition is reduced due to surface resurfacing or regeneration. Rise is the height of the average level of the ocean surface and the increase in wavelength around it. The mayfly optimization algorithm (MOA) was proposed with a better hybridization of the particle swarm optimization (PSO) and the differential evolution (DE) algorithms. The velocity would be relevant to the Cartesian distance among the relevant individuals.
\end{abstract}

Keywords: Mayfly algorithm, Multi-objective optimization, Optimization algorithm, Climate change

\section{Introduction}

Our results show significant change in Freshwater pests under climate change, according to the results of other studies, suggest that the appropriate area should be replaced within a century to prevent loss of height and loss of suitable habitat for higher altitudes. Possible habitat is caused by changing climatic factors. In this study, we found that average annual temperatures and rainfall and the minimum temperature of winter are important factors for species range predictions. These factors are most closely related to altitude (average annual temperature: annual rainfall: minimum winter temperature: data not shown). This high slope of climatic conditions that shape the distribution of the sample species illustrates the upward shift of suitable areas under GCC scenarios. Montana cold-stenothermic species are expected to observe the climate envelope upwards altitude. We assessed the impact of environmental change and the executives rehearse through various water temperature and layer estimations. Water temperature estimations are the normal yearly temperature, the most extreme yearly temperature, the base yearly temperature, the normal yearly temperature, the greatest yearly temperature and the base yearly temperature. To cover the gap in the literature, this paper proposes the ANFIS-based technique for blade pitch control of the WECS, which transcends traditional controllers. This paper also suggests a new strategy for creating a training and testing database for ANFIS controller, creating a training-appropriate database, and adjusting and testing its membership functions. For this reason, another enhancement calculation called the Mayfly Optimization Algorithm is utilized to precisely distinguish the ideal boundaries of the regulator. The proposed strategy is contrasted and different techniques in the writing to demonstrate its benefits. To test the viability and strength of the proposed based method, a few test situations are performed. The positive elements of the proposed regulator give superb execution against wind speed vacillations, load prerequisite varieties, boundary vulnerabilities and delay of correspondence channels. However, cluster formation, $\mathrm{CH}$ selection and re-clustering do not provide efficient solutions for data transfer between vehicles, and $\mathrm{CH}$ selection plays an important role in clustering algorithms. Optimization is the optimal solution among the various solutions in the solution space Is a technique of presenting. Therefore, an optimization applies to the selection of the best node of the algorithm. However, some upgrade algorithms take a considerable amount of time to integrate into each iteration during the $\mathrm{CH}$ exam May take. Therefore, the selection of optimization algorithms to determine network functions such as service and energy efficiency, the current study proposes the MADCR protocol to maximize the lifespan of networks and greatly reduce vehicle latency.

\section{Mayfly Algorithm}

In this context, a novel multi-objective Mayfly algorithm (MOMA) based on the MA and NFL was proposed, in which the archive algorithm, non-dominant sorting strategy and roulette wheel selection were added to further improve performance and integration performance. Algorithm. In solving many objective optimization problems. In this study, a new wind speed group forecasting system (WSEFS) was proposed based on data decay technology, sub-sample selection strategy, multiple predictive sub-models and the newly proposed MA, Point Forecast (PF). And interval forecast (IF). In particular, VMD is used to 

predictions are used. [1] Mayfly algorithm (MA) is an optimal way to achieve this goal. It is a newly developed algorithm that combines the key benefits of PSO, GA and FA, resulting in a hybrid of PSO-GA-FA. Compared with the other seven transformation methods, the performance evaluation performed using the CEC test operations demonstrates the dominance of MA in terms of accumulation rate and velocity. Although this requires rapid integration, it is not yet used in many engineering optimization fields, so we propose to use it for antenna optimization tasks. [2] The Mayfly algorithm is a new and efficient bio-inspired optimization technique that sometimes solves different optimization problems, and improving issues using the Mayfly algorithm provides a weaker solution than other upgrade algorithms. To improve the accuracy of this algorithm and solve the problem described, the local search program uses Elite Maple pairs for each iteration. The symmetric muffle algorithm (BMA) method for upgrading is given below. First, the elite male maple was found to have the lowest price value in the population. Then the number of seeds was selected. Later, female flies attract elite males to produce new offspring, hence the radius of growth space. To verify the effectiveness of a given BMA, it performed four test operations and compared its results with some new bio-train algorithms, including the Balance Optimizer. [3] Mayflies40 belongs to the genus Ephemeroptera, an ancient genus of polyoptera. The proposed MA optimization system is a powerful hybrid algorithm that Muffles are the backbone of the proposed algorithm for social behavior, especially considering the main advantages of their mating process: PSO, genetic algorithm and firefly algorithm. In the MA Mayfly article, various reports have suggested that the PSO algorithm may require some serious improvements with the help of various evolutionary guidelines and standards. Test bench functions, which confirm the reliability of MA more than other algorithms. That's why he earned his M.A.[4] Gained, the authors in this article use confusing mapping and natural learning resistances to hybridize MA to get the best results and confirm the truth. Mayflies are already considered adults, and no matter how long they live, qualified flies will survive after hatching from eggs. The position of each map in the search area indicates a possible solution to the proposed problem. The algorithm works as follows. Initially, approximately two sets of mafias were formed to represent the male and female population. In the specified algorithm, each muffler is located as a candidate solution in the approximate $\mathrm{N}$ dimensional problem search area, which is referred to as the vector. [5] Muffle is a transformation mechanism that is the result of a change in the Particle Mass Optimization (PSO) Algorithm Behavior. This is triggered by the social behavior of the Mayflies. Initially, bundles of files were created in approximate network clusters, which were used to evaluate the quality. the file at the fitness function solution location. According to the MADCR protocol, exercise performance is calculated based on the distance between moving vehicles during the gravity process. The best female muffler in terms of speed values attracts the best male muffler. The value of speed is updated based on the exercise activity [6] In this section, the proposed optimization of the radial-based functional neural network is described. MA is used to solve optimization problems, and it is triggered by flying behavior and the mating process of muffles. $37 \mathrm{RBFNN}$ is used as a radial based operational function, as well as an approximate function, time forecast classification and control system.[7] The key components of the system operate with investment, replacement and maintenance costs. Under certain optimization approaches GA, PSO, ant lion optimizer (ALO), dragonfly algorithm (DA) and other optimization algorithms are not suitable for delivering universal solution due to valve point impacts, restricted operating zones, and fragmented cost functionality. MDA has strong parallel search capability and gets local minimums. [8] This is the dynamic piece of the present exploration. The No Free Lunch (NFL) hypothesis expresses that the normal exhibition for all issues in a class is no different for any arrangement. This intends that in a specific issue, various calculations might get various outcomes, however in all issues they will be something similar. It urged analysts to make before work and concocted new issue explicit methodologies for further developed execution. In this paper, the creators have proposed a mixture calculation in view of the Harmony Search (HS) calculation and the Mayfly calculation (MA) to tackle the FS issue. The HS calculation has been utilized to update across different spaces previously. Mama is an as of late proposed meta-heuristic that has been demonstrated to be successful in managing streamlining issues [9].

\section{Multi-Objective Optimization}

These MOEAs, due to their demographic nature, can approximately the entire barreto-front (PF) of the MOP in a single flow. Also, as search location information is exchanged between search agents, they can quickly integrate with the actual Paretofront. The Strength Barretto Evolutionary Algorithm (SPEA) was proposed and Thiel stores the Barretto-optimized solutions he has discovered so far in an external archive. The algorithm uses Barreto Dominance to assign exercise values to individuals, and these exercise values are determined only from solutions that do not dominate the external archive. Then, it reduces the number of non-dominant solutions stored in the external archive without destroying it. barreto-lead properties. In SPEA, all solutions in the archive are selected and a new nickname is used that does not require any exercise sharing parameter. [10] The unique DV is related to the landscape change and a numerical process Proposed. This DV will remain stable until the algorithm integrates into a local optimization, after which the algorithm will restart and the optimization process will continue. In this problem, the domain may be concentrated and unconnected, and there is no guarantee of reaching the global minimum, so a multi-start process is used to find the best solution and the least expensive and safest solution is selected [11]. The user must create multiple initial solutions for the multiple startup process. Different initial geometry and sectional design variables were considered with pre-design formulas (e.g., towers were designed before total weight. members should not exceed fourquarters). [12] This survey refers to a series of non-linear multipurpose update methods. Section 2 lays the foundation for basic concepts. Then, since the primary goal of multipurpose optimization is to model the decision maker's preferences (sorting or comparative importance of materials and goals), the methods are categorized according to how the decision maker expresses these preferences. Section 3 contains methods that include the primary pronunciation of the options, which specify the objective functions or the relative importance of the desired goals before executing the optimization algorithm [13]. Section 4 describes the methods with pronunciation behind the options, which will select a single solution from a set of mathematically equivalent solutions. The pronunciation of the unwanted methods options is specified. Algorithms that include progressive pronunciation of options that the decision maker provides following input when the algorithm is running are not discussed. 
M. Amudha. et.al / Recent trends in Management and Commerce Vol: 2(3), 2022: 23-28

[14] The ultimate goal of the Multi-Objective Optimization Algorithm is to identify solutions within the Pareto optimal package. However, for many multipurpose issues, it is practically impossible to identify the optimal set for the entire Barreto due to its size. In addition, for many problems, especially integrated optimization problems, the evidence for solution improvement is computationally impossible. Therefore, the practical approach to multi-objective optimization is to explore as much as possible the set of solutions (the well-known Barreto suite) that represents the Barreto optimal set. With these concerns in mind, the multi-reference optimization approach should achieve the following three conflicting goals. [15] despite many initial attempts, Barreto's idea of hope in evolution was rejected by David E. Directly linked by Goldberg, first pointed out in his Seminal book on genetic algorithms. People expecting Barreto on the problem of multi-objective optimization. The basic idea is to find a set of solutions for non-Barreto people. These solutions will be assigned to the highest standards and removed from controversy. Another solution that Barreto does not dominate is determined by others and assigned to the next highest office. This process will continue until all people have received the appropriate rankings. Goldberg also suggested using some kind of nitching technique to avoid turning the GA into a point in front. [16] This paper proposes a new multipurpose optimization algorithm called Pareto-MEC. It introduces Barreto's theory in the MEC to improve a number of objective issues. Principles of Pareto-MEC: Many individuals are scattered throughout the entire settlement, and then some of the best individuals are selected as starting points for each group. Each group searches for only one local area and gradually shifts from its initial center to the Pareto front. During the conversion process to this first page, this algorithm controls the search area of the panel and changes the direction of the panel [17]. Both the above function and function are called dissimilation and the function is called homogeneous taxi. There are some differences from the basic MEC described in Pareto-MEC [18] we discuss various ways to systematically design experimental issues for multi-objective optimization: multi-objective approach, bottom-up approach and control-surface approach. The first approach was implicitly used by early MOEA researchers to create more intuitive and experimental problems. In this approach, M different single-purpose functions are used to create the multipurpose test problem. To facilitate the construction process, in many cases, different objective functions are used as different translations of an objective function[19]. For example, it is not optimal that SCHl uses to minimize the following two single-purpose functions, but rather the Pareto-optimal package contains more than one solution, which includes a unique minimum of each of the above functions. All other solutions that create exchanges between two objective functions become members of the list of many such experimental problems with the above two solutions. It is worth noting that such a construction process can extend to high vision problems, which can be difficult to understand in seemingly simple and complex issues [20].

\section{Optimization Algorithm}

This paper highlights the importance of shift variation for global optimization algorithms and as a test bed for WOAs in the performance of optimization algorithms in the absence of shift variation properties. Explores the impact of use. This attribute ensures that the performance of the optimization algorithm depends on the shape / form of a terrain; however, this does not depend on the location of the terrain you are looking for[36]. The ultimate goal of this research article Is to provide horticulturists and researchers with a guide to designing new methods keeping in mind the characteristics of shift variation. For example, Team Game Algorithm (DGA) editors used modified benchmark functions to evaluate the performance of their algorithm. [37] The emergence of more and more complex optimization problems leads to a greater demand For optimization algorithms. Over the past few years, researchers have explored a number of meat protocol guidelines, both for simplicity and implementation With the benefits of simplicity, competitive alternative resolution projects have been taken up to solve complex optimization problems[38]. These algorithms are primarily classified into four types of evolutionary algorithms (optimization algorithms based on different evolutionary and biogeography algorithms (including Ray-optimization algorithm and multi-algorithm) and mass intelligence algorithms. [39] To further study the Simulation results from the proposed algorithm show the I / V curves, which compare the voltage calculated by the model with the voltage measured experimentally. Provides. The exact match between the calculated and measured voltages is clearly visible. Furthermore, the characteristics presented in this figure reflect the accuracy and practicality of the model confirm the ability to evaluate results very closely to those seen. This table provides a detailed comparison of the proposed algorithm and other optimization algorithms such as WOA44 and GHO33. [40] The PSO system is based on mass intelligence. The study is at an early stage. Beyond the Gene Algorithm (GA) and Simulated Annealing (SA) approaches do not have a POS systematic calculation method and definite mathematical basis [41]. Currently, this method can only be used successfully in the aspect of the evolutionary neural network, and its other applications are still being explored. According to national documents, research on PSOs is primary Mathematical basics and applied research. The mathematical basis includes the mechanical theory of the PSO, proof of its synchronization and strength, and more. Commonly published documents include its mathematical basics, integration and speed evaluation includes some documentation about. Integration not available[42]. It seeks to complete research on PSO; Application research involves pursuing its advantages, overcoming its shortcomings, and improving its application. Limitations. Since the structured pseudo energies depend on the set of materials used in the optimization algorithm, it is important that the physics of the atom have relevant contexts. Also, with a maximum of two atoms per unit cell to reduce the calculation time [43]. We choose more symmetrical structures. Representatives of the metallic environment are a cube-cube structure centered on a cube and a cube face. Ionic environments are delivered in a rock-salt or zinc-compound system where their common antioxidants We attach the elements that take the position. This leads from the lithium group to the fluorine group, the beryllium group to the oxygen group and the composition of many elements. We always use three small components of the respective groups to guarantee the amount variation of the resulting compounds. To change metals, multiple oxidation states are often possible [44].

\section{Climate Change}

We determined the Epilimnian temperature as the normal temperature over the thermocline during the separation time frame and the normal temperature of the water during the blending time frame[48]. Additionally, we determined the hypoallergenic temperature beneath the mean temperature underneath the thermocline and the normal temperature of the water when 
blending. Layer estimations are the thermocline profundity, the beginning date of the layer time frame, the end date of the layer time frame and the length of the layer time frame. [49] When J-NABS was launched; some conservationists realized that man-made Climate change threatens biodiversity. However, within a few years, it appeared on the NABS agenda, and now the future conditions and protection of land, sea and freshwater ecosystems Dominates discussions about planning. Rising water temperatures appeared in the form of short-term ice and geological limitations or phonological changes in freshwater animals. Warm temperatures microorganisms[50], plants and outdoor hot water can directly affect the metabolism of animals, as well as organisms with short heat tolerance. Climate change caused by changes in the amount and timing of precipitation, evaporation and glacier melting can cause large water changes. More stagnant water will change, resulting in biodiversity changes. [51] Understanding the impacts of current worldwide environmental change are fundamental internationally as well as at the territorial level, as worldwide normal temperature rise and changes in precipitation designs are outrageous temperatures and Increase the recurrence of downpour occasions[52]. Two examples under IHA programming were utilized to investigate the effect of environmental change on the Kikuchi River and to evaluate water changes and EFCs. Expanding air temperature is one of the impacts of environmental change, and water temperature Changes are likewise examined in this study since it has long haul consequences for stream biological systems. Utilizing the SWAT model, we mimicked momentum and future every day waterway streams utilized as contributions for IHA programming that breaks down 38 boundaries. [53] We also analyze the rate of change of surface specific humidity under climate change. Since the vapor is often concentrated near the surface, it is entirely from the conversion rate of the column vapor Cannot be different, however, from the vertical variations in average temperature and humidity and their changes under global warming. Basically it does not work properly[54]. With the exception of higher latitudes in both hemispheres, the conversion rate of the surface specific humidity is generally smaller than that of the column vapor. Under climate change. Constant humidity and surface concentration at $50 \circ \mathrm{S}$ latitudes - Difference between transfer rates of column vapors with specific humidity $2 \% \mathrm{~K}$ - 1 . Change for surface and column sizes [55] On the one hand, we should not assume that local edemas that do not have current climate limits within Their range will be safe from future climate change. First, the short distribution of nests in some organisms may have led to evolutionary expertise in physiological properties in relatively stable climates over the past 10,000 years, so they may have been subject to climate change[56]. Hundreds of thousands They may argue the opposite as they have withstood climate change for years, but exhibiting the ability to withstand very cold 'glacier' temperatures does not necessarily have to be an indication that they will sustain new high temperatures. Second, these species did not initially respond to climate change because they were different Are defined by factors. But with further warming, abnormal ecosystem (emission) organisms can move faster than possible climatic conditions, causing a rapid population decline without the slightest forecast. Third, these species now live in ecosystems in which the identities of other organisms and relatively numerous changes have already changed as a result of climate change. [57] Interstate "Global sea level has risen over the last 100 years, and this rise may be related to an increase in global average temperature".

\section{Conclusion}

Predictions for the next century 15 to $95 \mathrm{Cm}$, the $50 \mathrm{~cm}$ 'best estimate' indicates a significant increase in sea level rise over the last century, with records from both the Atlantic and the Pacific in the Northern Hemisphere showing sea increase. However, in most cases, of some sites This condition is reduced due to surface resurfacing or regeneration. Rise is the height of the average level of the ocean surface and the increase in wavelength around it. In addition, there is a correlation between these two phenomena, which cannot be generalized, but depends on the local geography [58]. Our specific goals are to determine the number of lineages shared between Madagascar and Africa and what is the reason for any one region. The proposed technique is compared with various methods in the literature to prove its merits. To test the effectiveness and strength of the proposed ANFIS based technique, several test scenarios are performed. Evidence for the onset of man-induced climate change in freshwater has already emerged in the form of rising water temperatures, short-term glaciers and geological boundaries or phonological changes in freshwater animals. Warmer temperatures can directly affect the metabolism of microorganisms, plants and outdoor hot water animals, as well as organisms with short heat tolerance.

\section{Reference}

1. Liu, Zhenkun, Ping Jiang, Jianzhou Wang, and Lifang Zhang. "Ensemble forecasting system for short-term wind speed forecasting based on optimal sub-model selection and multi-objective version of mayfly optimization algorithm." Expert Systems with Applications 177 (2021): 114974.

2. Chen, Liang, Chunxiang Xu, Heqing Song, and Kittisak Jermsittiparsert. "Optimal sizing and sitting of EVCS in the distribution system using metaheuristics: A case study." Energy Reports 7 (2021): 208-217.

3. Rauf, Mohammad, and M. Z. M. Noman. "Socio-Legal Dimensions of Family Health under Marital Settings of Marriage and Divorce in the Maldives." European Journal of Molecular \& Clinical Medicine 7, no. 11 (2021): 5215 5221.

4. Majumdar, Kingsuk, Provas Kumar Roy, and Subrata Banerjee. "Implementation of multi-objective chaotic mayfly optimisation for hydro-thermal-solar-wind scheduling based on available transfer capability problem." International Transactions on Electrical Energy Systems 31, no. 11 (2021): e13029.

5. Sennan, Sankar, Somula Ramasubbareddy, Sathiyabhama Balasubramaniyam, Anand Nayyar, Chaker Abdelaziz Kerrache, and Muhammad Bilal. "MADCR: Mobility aware dynamic clustering-based routing protocol in Internet of Vehicles." China Communications 18, no. 7 (2021): 69-85.

6. Bhattacharyya, Trinav, Bitanu Chatterjee, Pawan Kumar Singh, Jin Hee Yoon, Zong Woo Geem, and Ram Sarkar. "Mayfly in harmony: A new hybrid meta-heuristic feature selection algorithm." IEEE Access 8 (2020): 195929195945. 
M. Amudha. et.al / Recent trends in Management and Commerce Vol: 2(3), 2022: 23-28

7. Das, Amit Kumar, Ankit Kumar Nikum, Siva Vignesh Krishnan, and Dilip Kumar Pratihar. "Multi-objective Bonobo Optimizer (MOBO): an intelligent heuristic for multi-criteria optimization." Knowledge and Information Systems 62, no. 11 (2020): 4407-4444.

8. Ramachandran, M., Sahas Bansal, and Pramod Raichurkar. "Scrutiny of jute fiber poly-lactic acid (PLA) resin reinforced polymeric composite." Journal of the Textile Association 76, no. 6 (2016): 372-375.

9. Konak, Abdullah, David W. Coit, and Alice E. Smith. "Multi-objective optimization using genetic algorithms: A tutorial." Reliability engineering \& system safety 91, no. 9 (2006): 992-1007.

10. Rauf, Mohammad. "Issues and Impact of COVID-19 on Socio-Legal Aspects of Human Life: A Case Study of Greater Male Region'in the Maldives." Asian Journal of Research in Business and Management 3, no. 1 (2021): 70 79.

11. Deb, Kalyanmoy, Lothar Thiele, Marco Laumanns, and Eckart Zitzler. "Scalable multi-objective optimization test problems." In Proceedings of the 2002 Congress on Evolutionary Computation. CEC'02 (Cat. No. 02TH8600), vol. 1, pp. 825-830. IEEE, 2002.

12. Kumar, M. Senthil, and P. Sivakumar. "Need For Curriculum Reforms In Technical Education To Achieve Outcome Based Education."

13. Sharma, Deepa, and DR C. VENKATESWARAN. "Discrimination Face Female Faculty During the Recruitment \& Selection and Training Time in The Academic Sector." Journal of Contemporary Issues in Business and Government 27, no. 3 (2021): 1104-1108.

14. Kalita, Kanak, Uvaraja Ragavendran, Manickam Ramachandran, and Akash Kumar Bhoi. "Weighted sum multiobjective optimization of skew composite laminates." Structural Engineering and Mechanics 69, no. 1 (2019): 21-31.

15. Monaghan, Michael T., Jean-Luc Gattolliat, Michel Sartori, Jean-Marc Elouard, Helen James, Pascale Derleth, Olivier Glaizot, Ferdy de Moor, and Alfried P. Vogler. "Trans-oceanic and endemic origins of the small minnow mayflies (Ephemeroptera, Baetidae) of Madagascar." Proceedings of the Royal Society B: Biological Sciences 272 , no. 1574 (2005): 1829-1836.

16. Kumar, R. Dinesh, C. Sridhathan, and M. Senthil Kumar. "Performance Evaluation of Different Neural Network Classifiers for Sanskrit Character Recognition." In Business Intelligence for Enterprise Internet of Things, pp. 185194. Springer, Cham, 2020.

17. Whittaker, Robert J., Miguel B. Araújo, Paul Jepson, Richard J. Ladle, James EM Watson, and Katherine J. Willis. "Conservation biogeography: assessment and prospect." Diversity and distributions 11, no. 1 (2005): 3-23.

18. Manickam, Ramachandran. "Back propagation neural network for prediction of some shell moulding parameters." Periodica Polytechnica Mechanical Engineering 60, no. 4 (2016): 203-208.

19. Simon, Michael J., and Mark A. Aitken. "Next generation terrestrial broadcasting platform aligned internet and towards emerging 5G network architectures." U.S. Patent 10,652,624, issued May 12, 2020.

20. Gadde Mehar Chaitanya, M.P.Jenarthanan, C. Sathiyaraj, A Review on Glass fibre Reinforced Composites with Different Matrix, REST Journal on Emerging trends in Modelling and Manufacturing Vol: 7(1), 2021, 18-24.

21. Shameem, Ahmad, and Mohammad Rauf. "Factors Influencing the Implementation of Remote Learning in Higher Educational Institutions in Male" City during COVID-19 Pandemic." COVID-19 emerging socio-economic and legal trends in Maldives.(pp-80-97). Mahi Publication Pvt. India (2021).

22. Venkateswaran, Dr C. "Family Responsibilities Make a Barrier in the Career of Female Faculty." Mrs. Deepa Sharma, Dr. C. Venkateswaran." Family Responsibilities Make a Barrier in the Career of Female Faculty". International Journal of Computer Engineering In Research Trends (IJCERT), ISSN (2020): 2349-7084.

23. Askari, Qamar, Irfan Younas, and Mehreen Saeed. "Emphasizing the importance of shift invariance in metaheuristics by using whale optimization algorithm as a test bed." Soft Computing 25, no. 22 (2021): 14209-14225.

24. Lokhande, Dr Amol, Dr C. Venkateswaran, Dr M. Ramachandran, C. Vidhya, and R. Kurinjimalar. "A Study on Various Implications on Reusing in Manufacturing." REST Journal on Emerging trends in Modelling and Manufacturing 7, no. 2 (2021).

25. Jiang, Ping, Zhenkun Liu, Jianzhou Wang, and Lifang Zhang. "Decomposition-selection-ensemble forecasting system for energy futures price forecasting based on multi-objective version of chaos game optimization algorithm." Resources Policy 73 (2021): 102234.

26. Shaheen, Mohamed AM, Hany M. Hasanien, M. S. El Moursi, and Attia A. El-Fergany. "Precise modeling of PEM fuel cell using improved chaotic MayFly optimization algorithm." International Journal of Energy Research 45, no. 13 (2021): 18754-18769.

27. Sathiyaraj Chinnasamy, M. Ramachandran, Kurinjimalar Ramu, P. Anusuya "Study on Fuzzy ELECTRE Method with Various Methodologies" REST Journal on Emerging Trends in Modelling and Manufacturing, 7(4),(2021),108115

28. Prats, Jordi, Marie-Jose Salencon, Magali Gant, and Pierre-Alain Danis. "Simulation of the hydrodynamic behaviour of a Mediterranean reservoir under different climate change and management scenarios." J. Limnol 77 (2018): 62-81.

29. Kaur, Mandeep, and Dr C. Venkateswaran. "To Study the Work Life Balance among Working Women, Post Maternity in Banking Sector." International Journal of Management (IJM) 11, no. 2 (2020).

30. Morid, Reihaneh, Yukihiro Shimatani, and Tatsuro Sato. "Impact assessment of climate change on environmental flow component and water temperature-Kikuchi River." Journal of Ecohydraulics 4, no. 2 (2019): 88-105.

31. Godbole, Nishant, Shajit Yadav, M. Ramachandran, and Sateesh Belemkar. "A review on surface treatment of stainless steel orthopedic implants." Int J Pharm Sci Rev Res 36, no. 1 (2016): 190-4.

32. Richards, Christopher T., and Christofer J. Clemente. "A bio-robotic platform for integrating internal and external mechanics during muscle-powered swimming." Bioinspiration \& biomimetics 7, no. 1 (2012): 016010. 
M. Amudha. et.al / Recent trends in Management and Commerce Vol: 2(3), 2022: 23-28

33. Dr. Amol Lokhande, Dr. C. Venkateswaran, Dr. M. Ramachandran, C. Sathiyaraj, K. Nathiya, Recycling Process Impact in Current Scenario Manufacturing: A Study, Recent trends in Management and Commerce Vol: 2(1), 2021, 20-25.

34. Nomani, M. Z. M., Ajaz Afzal Lone, and MOHAMMAD RAUF. "Prevention of mental health disorders among women and children of kashmir valley: a socio-legal perspective." International Journal of Pharmaceutical Research 12 , no. 2 (2020).

35. Soniya Sriram, M. Ramachandran, Sathiyaraj Chinnasamy, G. Mathivanan “A Review on Multi-Criteria DecisionMaking and Its Application” REST Journal on Emerging Trends in Modelling and Manufacturing, 7(4), (2021), 101107

36. Short, Frederick T., and Hilary A. Neckles. "The effects of global climate change on seagrasses." Aquatic Botany 63, no. 3-4 (1999): 169-196.

37. Nair, Vineeth, Pratul Khosla, and M. Ramachandran. "Review on mechanical properties of various natural fibers reinforced composites." Research Journal of Pharmaceutical Biological and Chemical Sciences 7, no. 1 (2016): 2001 2004.

38. Katakam, Lakshmi Narasimha Rao, Thirupathi Dongala, and Santhosh Kumar Ettaboina. "Novel stability indicating UHPLC method development and validation for simultaneous quantification of hydrocortisone acetate, pramoxine hydrochloride, potassium sorbate and sorbic acid in topical cream formulation." Talanta Open 1 (2020): 100004.

39. Yenda, Parvateesam, Naresh Kumar Katari, Thirupathi Dongala, Govind Vyas, Lakshmi Narasimha Rao Katakam, and Santhosh Kumar Ettaboina. "A simple isocratic LC method for quantification of trace-level inorganic degradation impurities (ferricyanide, ferrocyanide, nitrite, and nitrate) in sodium nitroprusside injection and robustness by quality using design approach." Biomedical Chromatography 36, no. 2 (2022): e5269.

40. Katakam, Lakshmi Narasimha Rao, Thirupathi Dongala, and Santhosh Kumar Ettaboina. "Quality by design with design of experiments approach for development of a stability-indicating LC method for enzalutamide and its impurities in soft gel dosage formulation." Biomedical Chromatography 35, no. 5 (2021): e5062.

41. Nomani, M. Z. M., Faizanur Rahman, Mohammad Rauf, and Saif A. Khan. "Access and Benefit Sharing Models of Biodiversity Conservation in International and Comparative Law Perspective." Advances in Natural and Applied Sciences 14, no. 1 (2020): 1-14.

42. Pradeep, P., J. Edwin Raja Dhas, M. Ramachandran, and B. Stanly Jones Retnam. "Mechanical Characterization of jute fiber over glass and carbon fiber reinforced polymer composites." International Journal of Applied Engineering Research 10, no. 11 (2015): 10392-10396.

43. Sridhathan, Senthilkumar, and M. Senthil Kumar. "Plant Infection Detection Using Image Processing." International Journal of Modern Engineering Research (IJMER) 8 (2018).

44. Lokhande, Dr Amol, Dr C. Venkateswaran, Dr M. Ramachandran, S. Chinnasami, and T. Vennila. "A Review on Various Implications on Re engineering in Manufacturing." REST Journal on Emerging trends in Modelling and Manufacturing 7, no. 3 (2021): 70-75. 